\title{
Parametric representation of helioseismic data
}

\section{A method for estimation of mode correlation}

\author{
A. Ferrari ${ }^{1,2}$, F.-X. Schmider ${ }^{1}$, A. Alengrin ${ }^{1,2}$, and B. Gelly ${ }^{1}$ \\ 1 UMR 6525 Astrophysique, Université de Nice-Sophia-Antipolis, F-06108 Nice Cedex 2, France \\ 2 I3S UPRES-A 6070, Université de Nice-Sophia-Antipolis, F-06560 Valbonne, France
}

Received June 16, 1998; accepted March 12, 1999

\begin{abstract}
This paper describes a parametric method aimed at the estimation of correlation in the excitation of solar $p$ modes. Unlike previous work which investigated the correlation in the statistical properties of the mode's power, rather we study the excitation signal itself. In this approach, each mode is represented as a second-order autoregressive process and the correlation of the modes is modeled by the covariance of the excitation inputs. We have developed an algorithm to estimate in two steps the dynamical parameters of the modes and the covariance matrix of the excitation. We first extract the denominator of the ARMA process resulting from the sum of the different modes, then estimate iteratively the covariance matrix of the excitation.

The method has been first validated on simulated signals. We verified that the power spectrum of an $l=1$ mode is modified by the existence of correlation in the excitation when the modes overlap. This leads to an incorrect estimation of the rotational splitting when assuming independence of the components. We checked the ability of our method to recover the correlation of the excitation and the true frequencies. We also applied the method to real data. The results have been compared to the traditional Lorentz fitting. For the $l=1$ modes, a good agreement on the parameters of the modes has been found, although no constraints have been imposed on the amplitudes and widths. In the present paper, we develop the mathematics of the method, and present the results obtained on simulations, as well as an application to the GOLF data. The results about correlation in the excitation of solar $p$ modes will be reported in a forthcoming paper.
\end{abstract}

Key words: solar oscillations — data analysis

Send offprint requests to : F.-X. Schmider

\section{Introduction}

Solar $p$ modes are acoustic waves generated by the convection and trapped between the solar surface and an inner turning point, leading to a resonance at given frequencies. Those modes are typically represented by a damped harmonic oscillator excited by a stochastic process:

$\ddot{x}+2 \Gamma \dot{x}+\omega_{0}^{2} x=F$

where $F$ is a Gaussian noise, $\omega_{0}$ the frequency and $\Gamma$ the damping rate of the mode. In the Fourier domain, the result is approximated by a lorentzian shape which can be fitted by Maximum Likelihood Method to the data periodogram, taking into account its asymptotic independent $\chi^{2}$ distribution (Brillinger 1981; Toutain \& Appourchaux 1994; Fierry-Fraillon et al. 1998). This method is generally used to extract the mean parameters of the modes (amplitude, width, frequency). Nevertheless, it suffers limitations, as it does not take into account effects of the excitation, such as the location and extent of the excitation source, which produces asymmetries of the line profile (Gabriel 1995; Roxburgh \& Vorontsov 1995). Moreover it assumes that the modes are independent, which has never been demonstrated.

In fact, little is known about the convection. The coupling between convection and oscillations is generally described using so-called "mixing-length theory", for which several free parameters remain, such as the ratio of entropy fluctuation to Reynold stress, a ratio of horizontal vs vertical motion of the convective eddies, or parameter describing the small scale structure of the turbulent spectrum (Peter Goldreich \& Kumar 1994; Balmforth 1992). However, there are few observational constraints (Kumar 1997). Save for the above mentioned asymmetries (which have not yet been measured on low-degree modes) and the study of the pseudo-modes, which test both the location and size of the excitation source, the only observables containing information on the convection are the statistical properties of the mode amplitude and damping. 
The values obtained by Lorentz fitting are mean values, but it would be useful to test the higher order statistic properties.

Our idea is to improve the mathematical representation of the modes in order to address the effects of convection. For this we explore the possibilities of a parametric model. This representation is well adapted to studying the excitation signal itself. In principle, it would be possible to introduce any kind of effect included in the theoretical description, but with the drawback of increasing the complexity and the number of free parameters, thus making it difficult to solve. Therefore, we focused on looking for possible correlations in the mode excitation, keeping other hypothesis which appear to be justified for the solution of the present problem: the modes are still represented as solutions of a linear second order differential equation, and the possible temporal correlation of the noise source is neglected (this will be justified later).

\section{Modes correlation}

\subsection{Scientific context}

Several authors have studied the temporal behaviour and the statistical distribution of modes amplitude or power, using time-frequency analysis. The distribution has been found to follow mainly an exponential behaviour (Baudin et al. 1996; Foglizzo et al. 1998), in agreement with a Boltzman distribution due to a stochastic excitation (Kumar 1997). Although important for further data analysis, this does not constrain the description of the excitation. Nevertheless, some discrepancies remain in the high amplitude peaks (Gavryusev \& Gavryuseva 1997; Chaplin et al. 1997) which could have a non-stochastic behaviour, but the statistics are rather poor and deserve some strengthening.

Several tentatives have been made already to measure the correlation between modes (Foglizzo et al. 1998), by looking at the temporal behaviour of the mode power. The method consists in analysing the integral of the power in a given bandwidth, so it is intrinsically limited to the study of correlation between modes well separated in frequency (namely the $l=0$ modes). They have found no evidence for correlation in the GOLF signal.

As pointed out by the authors, no significative correlation between the power of the modes should be found even if there are excited by the same noise source (because they have different frequencies), unless the excitation itself were to have a temporal correlation longer that the life-time of the modes.

We checked by a simulation that this correlation is almost zero when the modes do not overlap and when the temporal correlation of the excitation is shorter than the life-time of the modes. Therefore, the result from Foglizzo et al. is not contradictory with an excitation of the modes by a common signal, but suggests that in this case, excitation by a common source requires a temporal correlation shorter that the life-time of the mode.

\subsection{Interest of the present method}

We have developed a completely different method, based on a parametric model, which allows us to extract the profile of the modes as with the lorentzian fit, but can also recover the characteristics of the excitation signal. Unlike the method described above, this allows us to study correlation in the excitation of modes which are very close in frequency, such as the modes split by rotation.

This can be very useful in order to improve our description of the coupling between convection and oscillations. Indeed, the free parameters in the model will have geometrical effects on non-radial modes, that could be addressed if the correlation in the signal exciting different modes with the same or different geometries could be measured.

It is also of fundamental importance for the measurement of the internal rotation: the frequencies separation between modes of the same degree and order (the rotational splitting) is measured with the assumption that the modes are uncorrelated. If this hypothesis is not correct, the value of the splitting could be affected by a bias, which could modify drastically the rotation rate (see Sect. 4.1).

\section{Mathematical development}

\subsection{Signal model}

The signal that we propose to analyse is the full-disk measurement from GOLF (Gabriel et al. 1995). It is regularly sampled and does not present any gaps. This signal is well known (Lazrek et al. 1997) for containing $l=0$ to 3 modes and marginally $l=4$ and $l=5$. Higher degree modes are averaged and remain, in particular in the lower frequency range, well below the noise level.

The signal is down-sampled to $80 \mathrm{~s}$ after being filtered above $12 \mathrm{MHz}$ to avoid aliasing. A small bandwidth of $\sim 6 \mu \mathrm{Hz}$ around an $l=1$ mode contains only a known number of modes. The wings of the modes surrounding the bandwidth studied can be regarded as an additional white noise.

It can be easily demonstrated that when $T_{\mathrm{s}} \nu_{0} \ll 1$ where $T_{\mathrm{s}}$ is the sampling frequency, the samples from the continuous model (1) verify a second order autoregressive equation:

$x_{n}^{(k)}=-a_{1}^{(k)} x_{n-1}^{(k)}-a_{2}^{(k)} x_{n-2}^{(k)}+e_{n}^{(k)}$,

where $e_{n}^{(k)}$ is a Gaussian white noise excitation. The index $k$ references the mode. This can be proved using the filter representation of the process and converting the analog 
filter to a discrete filter using the backward approximation. Various statistical justifications of this model can be found for example in (Priestley 1994).

It is worth noting that the width of the analysed frequency bands corresponds to an innovation rate of 2 days. The hypothesis of temporal independence of the excitation signal $e_{n}^{(k)}$ is justified by statistical analysis of the mode power already mentioned in Sect. 2 and in particular by the absence of correlation found by Foglizzo et al.

Finally, we assume that the measured signal $y_{n}$ is the sum of $L$ modes:

$y_{n}=\sum_{k=1}^{L} x_{n}^{(k)}+w_{n}, n=0, \ldots, N-1$,

and a Gaussian white noise with variance $\sigma^{2}$. We will assume herein that $\sigma^{2}$ is known. The set of measurements will be referred $\mathcal{Y}_{N}=\left\{y_{0}, \ldots, y_{N-1}\right\}$.

In the model $(2,3)$ the correlation between the different modes excitations is introduced by the covariance of the input terms. Let $\boldsymbol{E}_{n}=\left(e_{n}^{1}, \ldots e_{n}^{L}\right)^{t}$ be the vector of excitation and $Q$ its covariance: $E\left[\boldsymbol{E}_{n} \boldsymbol{E}_{n}^{t}\right]=Q$. The correlation coefficient between excitation of mode $i$ and $j$ is defined by $r_{i j}=Q_{i j} / \sqrt{Q_{i i} Q_{j j}}$.

The problem consists in the estimation of the coefficients of the autoregressive process, denoted $\theta$, and the covariance matrix $Q$.

\subsection{Position of the problem}

An immediate solution is to resort to maximum likelihood estimation. The process $y_{n}$ is Gaussian with covariance matrix $R_{y, N}(\theta, Q)$. Consequently, the estimated parameters are the global maximisers of the log-likelihood function or equivalently, after dropping the constant terms, of the function:

$\mathcal{L}\left(\mathcal{Y}_{N} ; \theta, Q\right)=-\log \left|R_{y, N}(\theta, Q)\right|-Y_{N} R_{y, N}(\theta, Q)^{-1} Y_{N}^{t},(4)$

with $Y_{N}=\left(y_{0}, \ldots, y_{N-1}\right)$. Unfortunately, the maximisation of (4) by an optimisation algorithm is not tractable because:

- the dimension $N \times N$ of $R_{y, N}(\theta, Q)$ leads to a large computational cost for the evaluation of $\mathcal{L}(\theta, Q)$ and the non-linear dependence between the unknown parameters and $R_{y, N}(\theta, Q)$ leads to a cost function that is highly non-linear, see Appendix A.

- the maximisation must be performed under the constraints that the autoregressive models are stable and the matrix $Q$ is semi-positive definite. Whereas the first constraint can be removed by a proper reparametrisation of $\theta$, a simple expression of the second constraint is not available.

A suboptimal solution is to minimise a distance between a $M$ order matrix $R_{y, M}(\theta, Q)$ and its estimated version $\hat{R}_{y, M}$, computed from the $y_{n}$. This solution significantly reduces the computational cost. If the cost function is defined as:

$\mathcal{C}(\theta, Q)=\operatorname{trace}\left(R_{y, M}(\theta, Q)-\hat{R}_{y, M}\right)^{2}$

it can be shown that its dependence on the elements of $Q$ is quadratic, see appendix A. However, the problems related to the nonlinear dependence on $\theta$ and the constraint on $Q$ remain.

A major difficulty in this estimation problem comes from the coupling between the two sets of parameter $\theta$ and $Q$. To address this, we propose a two stage approach that first estimates $\theta$ and then uses this result for the estimation of $Q$. Besides the different nature of $\theta$ and $Q$, this solution can be justified by the strong a priori that is available on the shape of the modes that can be incorporated in the first stage. Another argument is given by an analogy with the pure autoregressive case where the power of the excitation is generally computed from the autoregressive parameters and the data, (Stoica \& Moses 1997).

\subsection{Estimation of the dynamical parameters}

For this goal, we will derive a new statistical representation of $y_{n}$, i.e. a signal $\bar{y}_{n}$ with the same autocorrelation sequence as $y_{n}$. Consider the signal $z_{n}$ obtained by filtering $y_{n}$ :

$H(z)=\prod_{q=1}^{L}\left(1+a_{1}^{(q)} z^{-1}+a_{2}^{(q)} z^{-2}\right)=\sum_{q=0}^{2 L} c_{q} z^{-q}$.

Hypothesis $(2,3)$ implies that $z_{n}$ is the sum of each mode filtered by $H(z)$ and can be expressed:

$$
\begin{aligned}
z_{n} & =\sum_{k=1}^{L} H(z) x_{n}^{(k)}+H(z) w_{n} \\
& =\sum_{k=1}^{L} \sum_{q=0}^{2(L-1)} b_{q}^{(k)} e_{n-q}^{(k)}+\sum_{q=1}^{2 L} c_{q} w_{n-q}
\end{aligned}
$$

with:

$\sum_{q=0}^{2(L-1)} b_{q}^{(k)} z^{-k}=\prod_{q=1, q \neq k}^{L}\left(1+a_{1}^{(q)} z^{-1}+a_{2}^{(q)} z^{-2}\right)$.

Equation (8) implies $E z_{n} z_{n+l}=0$ for $|l|>2 L$. Then $z_{n}$ has a moving average representation of order $2 L$, (Stoica \& Moses 1997, p. 102):

$\bar{z}_{n}=A(z) \bar{e}_{n}, \quad A(z)=\sum_{k=1}^{2 L} b_{k} z^{-k}$.

Consequently $y_{n}$ has an autoregressive moving average representation where the coefficients of the autoregressive part equal the coefficients of $H(z)$ :

$\bar{y}_{n}=\frac{A(z)}{H(z)} \bar{e}_{n}$.

This result suggests the following algorithm for the 
estimation of the parameter vector $\theta$ :

1. Apply to $y_{n}$ an ARMA estimation algorithm in order to estimate its autoregressive part, $H(z)$.

2. Compute the filters $1+a_{1}^{(k)} z^{-1}+a_{2}^{(k)} z^{-2}$ combining the $L$ pairs of $H(z)$ conjugate roots.

We propose to estimate $H(z)$ using the subspace parameter algorithm described in (Stoica \& Moses 1997, p. 115). This method is based on the singular value decomposition of the estimated Block-Hankel matrix of covariances $\hat{C}_{y, M}$ whose element $(i, j)$ are the estimated autocorrelations $y_{n}$ at lag $|i+j-1|$. Let $\hat{\Sigma}$ the diagonal matrix made from the $2 L$ principal singular values of $\hat{C}_{y, M}$ and $\hat{U}$ the matrix containing the associated $2 L$ left singular vectors. Let $\bar{\Gamma}$ and $\underline{\Gamma}$ be the matrices made from the first and last, $M-1$ rows of $\hat{U} \hat{\Sigma}^{1 / 2}$. The roots of $H(z)$ are given by the eigenvalues of matrix $\Phi$, the least square solution of the system $\bar{\Gamma} \Phi=\underline{\Gamma}$.

\subsection{Estimation of the excitation covariance}

The second step of the method consists in replacing $\theta$ by its estimated value $\hat{\theta}$ and estimating the covariance of the vector $\boldsymbol{E}_{n}$. The derivation of an efficient algorithm for this requires a signal model that explicitly involves this vector process. A solution is to use a state space representation of $y_{n}$.

It can be easily verified that, up to a shift in the excitation, each mode can be represented by the following state space representation:

$\boldsymbol{X}_{n+1}^{(k)}=F^{(k)} \boldsymbol{X}_{n}+G^{(k)} \boldsymbol{E}_{n}^{(k)}, \quad x_{n}^{(k)}=H^{(k)^{t}} \boldsymbol{X}_{n}$.

with:

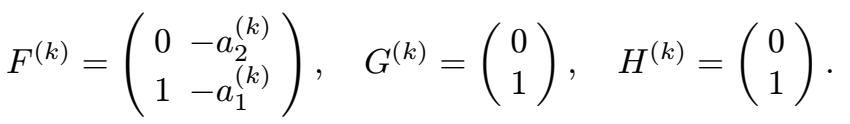

Consequently, a state space representation of $y_{n}$ is obtained by the concatenation of the previous models:

$\boldsymbol{X}_{n+1}=F \boldsymbol{X}_{n}+G \boldsymbol{E}_{n}, \quad y_{n}=H^{t} \boldsymbol{X}_{n}+w_{n}$,

where $F$ is a $2 L \times 2 L$ block diagonal matrix with the matrices $F^{(k)}$ on the diagonal. $H$ is the $2 L \times 1$ vector obtained by concatenation of the $H^{(k)}$, and $G$ is the $2 L \times L$ matrix having as non-zero elements $G(2 k, 2)=1$, $k=1, \ldots, L$. The unobserved set of states will be referred $\mathcal{X}_{N}=\left\{\boldsymbol{X}_{0}, \ldots, \boldsymbol{X}_{N-1}\right\}$.

It is worth noting that the model (13), contrary to the model $(2,3)$, allows a simple expression of the power spectrum density (PSD), (Anderson \& Moore 1979, p. 75): $S_{y}(\omega)=$

$\left[H^{t}(z I-F)^{-1} G Q G^{t}\left(z^{-1} I-F^{t}\right)^{-1} H+\sigma^{2}\right]_{z=e^{j \omega}}$.

As suggested in Sect. 3.1, we intend to estimate $Q$ as the global maximiser of $\mathcal{L}\left(\mathcal{Y}_{N}, \hat{\theta}, Q\right)$. The dependence on $\hat{\theta}$ will be dropped in the the following. The observation equation of model (13) shows that there exist a many-toone mapping between $\mathcal{X}_{N}$ and $\mathcal{Y}_{N}$. This suggests maximizing the likelihood by an iterative technique: the expectation maximisation algorithm (EM). This method is direct maximising the likelihood of $\mathcal{Y}_{N}$, the "incomplete data" making an essential use of $\mathcal{X}_{N}$, the "complete data". The reference paper on EM is Dempster et al. (1977) Moon (1996) has presented a tutorial on the EM algorithm including extensive references.

This algorithm has been recently applied in Deng and Shen (1997) for the estimation of all the parameters of a linear dynamic system. We will derive here the method for the case where the only unknown is $Q$.

The $E$ step is the computation of the conditional expectation of $\mathcal{L}\left(\mathcal{X}_{N} ; Q\right)$ given $\mathcal{Y}_{N}$ and the current estimate of $Q$, noted $\hat{Q}^{[k]}$. For the M step, let $\hat{Q}^{[k+1]}$ be that value of $Q$ that maximizes the previous conditional expectation.

In our case, the log likelihood of $\mathcal{X}_{N}$ is calculated using the Markov property of (12), (Anderson \& Moore 1979). Dropping all the terms that are not functions of $Q$, we have:

$\mathcal{L}\left(\mathcal{X}_{N} ; Q\right)=-\frac{N-1}{2} \log |Q|-\frac{1}{2} \sum_{k=1}^{N-1} G^{t} \boldsymbol{V}_{k}^{t} Q^{-1} \boldsymbol{V}_{k} G$,

$\boldsymbol{V}_{k}=\boldsymbol{X}_{k}-F \boldsymbol{X}_{k-1}$

Taking the conditional expectation of this equality and using the differentiation rules $\mathrm{d} \log |Q| / \mathrm{d} Q=Q^{-1}$ and $\mathrm{d} \boldsymbol{U}^{t} Q^{-1} \boldsymbol{U} / \mathrm{d} Q=-Q^{-1} \boldsymbol{U} \boldsymbol{U}^{t} Q^{-1}$ gives the following recursion for $\hat{Q}^{[k]}$ :

$\hat{Q}^{[k+1]}=\frac{1}{N-1} \sum_{k=1}^{N-1} G^{t} E\left[\boldsymbol{V}_{k} \boldsymbol{V}_{k}^{t} / \mathcal{Y}_{N}, \hat{Q}^{[k]}\right] G$.

Expansion of the inner term shows that each iteration requires the computation of terms $E\left[\boldsymbol{X}_{k} \boldsymbol{X}_{k}^{t} / \mathcal{Y}_{N}, \hat{Q}^{[k]}\right]$ and $E\left[\boldsymbol{X}_{k} \boldsymbol{X}_{k-1}^{t} / \mathcal{Y}_{N}, \hat{Q}^{[k]}\right]$. If $\hat{\boldsymbol{X}}_{k / n}$ is the conditional expectation of the state with respect to the observation and $\hat{Q}^{[k]}, P_{k / N}$ its variance and $P_{k, k-1 / N}$ the cross covariance between $\hat{\boldsymbol{X}}_{k / n}$ and $\hat{\boldsymbol{X}}_{k-1 / n}$, we have:

$E\left[\boldsymbol{X}_{k} \boldsymbol{X}_{k}^{t} / \mathcal{Y}_{N}, \hat{Q}^{[k]}\right]=P_{k / N}+\hat{\boldsymbol{X}}_{k / N} \hat{\boldsymbol{X}}_{k / N}^{t}$,

$E\left[\boldsymbol{X}_{k} \boldsymbol{X}_{k-1}^{t} / \mathcal{Y}_{N}, \hat{Q}^{[k]}\right]=P_{k, k-1 / N}+\hat{\boldsymbol{X}}_{k / N} \hat{\boldsymbol{X}}_{k-1 / N}^{t}$.

These quantities can then be computed, as in Deng \& Shen (1997), using a fixed interval smoothing algorithm, (Anderson \& Moore 1979) whose equations are summarized in Appendix B.

\subsection{Remarks on the algorithm}

- The development of Sect. 3.3 can be easily extended to incorporate the iterative estimation of $\sigma^{2}$. This solution has not been investigated here because of the high signal to noise ratio that has been observed in the analysed frequency band. In our case $\sigma^{2}$ has been estimated by the average of the bins of the periodogram surrounding the modes. 


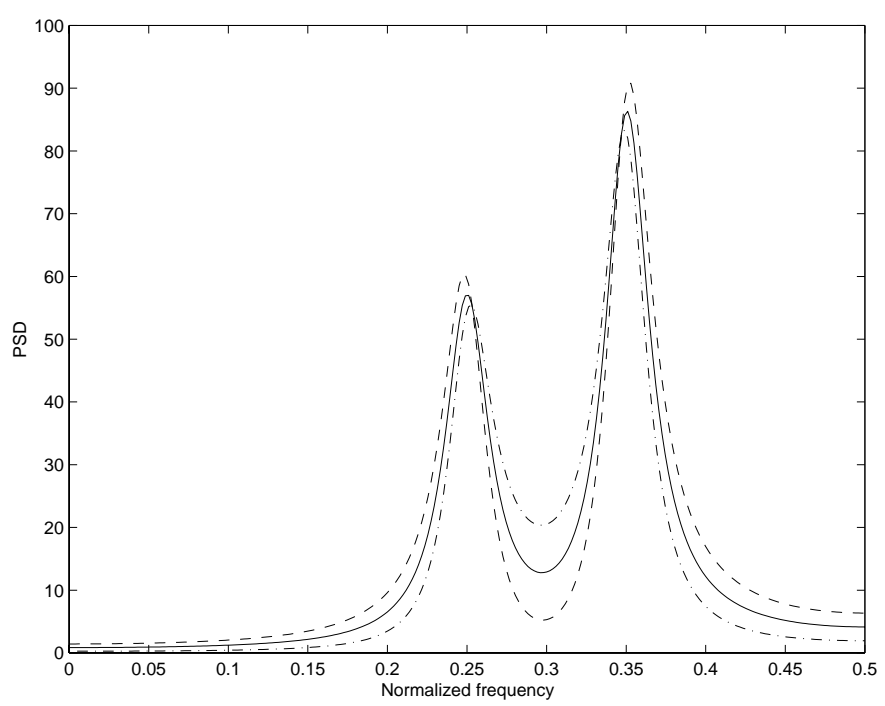

Fig. 1. Effect of $Q$ on the PSD. $Q_{11}=Q_{22}=2$. Continuous line $(-): r_{12}=0$, dashed line $(--): r_{12}=3 / 4$, dashed-dotted line $(-\cdot): r_{12}=-3 / 4$

- The structure of (15) guarantees that $\hat{Q}^{[k]}$ is semipositive definite.

- In Sage \& Melsa (1971), it is shown that the maximum a posteriori estimator of $Q$ is:

$$
\begin{aligned}
& \hat{Q}_{\mathrm{MAP}}=\frac{1}{N-1} \sum_{k=1}^{N-1} G^{t} \hat{\boldsymbol{V}}_{k / N} \hat{\boldsymbol{V}}_{k / N}^{t} G, \\
& \hat{\boldsymbol{V}}_{k / N}=\hat{\boldsymbol{X}}_{k / N}-F \hat{\boldsymbol{X}}_{k-1 / N} .
\end{aligned}
$$

An iterative implementation of this estimator can be obtained estimating the smoothed state vector at iteration $k$ using $\hat{Q}_{\mathrm{MAP}}^{[k]}$ and compute $\hat{Q}_{\mathrm{MAP}}^{[k+1]}$ using Eq. (16). It is worthy noting that this algorithm corresponds to the EM algorithm where the update of $Q$ is computed assuming that the matrices $P_{k / N}$ and $P_{k, k-1 / N}$ equal zero. A major drawback of this solution is that it does not guarantee an increase of the likelihood at each iteration.

- A large part of the previous development can be easily extended to the case where the modes are represented by autoregressive models of order higher than two, allowing the relaxation of the hypothesis of temporal independence of the noise input. The major difficulty that arises is to group the roots of $H(z)$ corresponding to the different modes.

\section{Computer experiments}

\subsection{Simulated signal}

We will now present three different numerical simulations. The first one shows the effect of a correlation on the power spectrum and its consequences on the measurement of frequencies. The second permits to verify the convergence
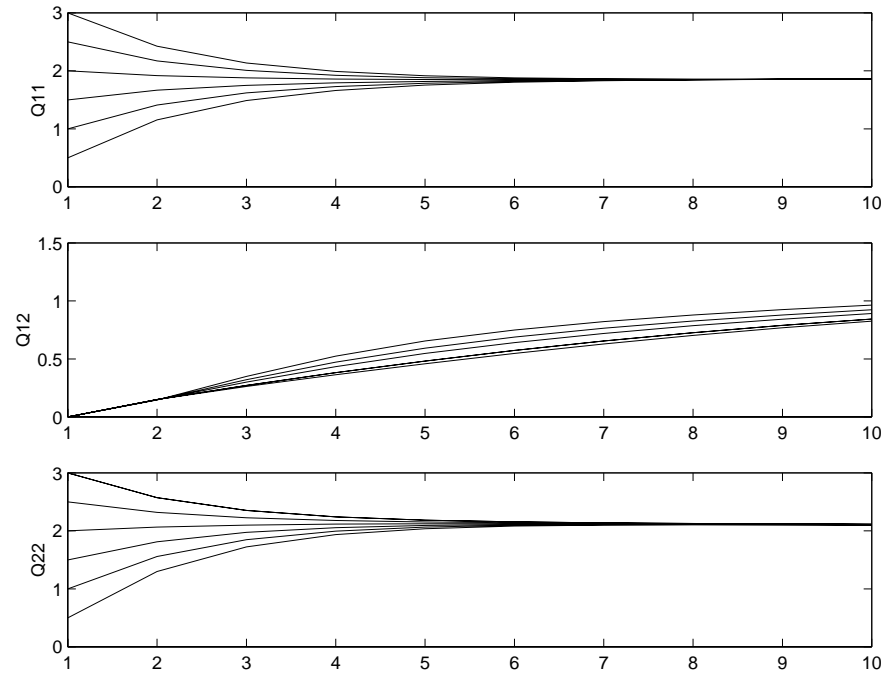

Fig. 2. Convergence of $\hat{Q}^{[k]}$ for different initial values

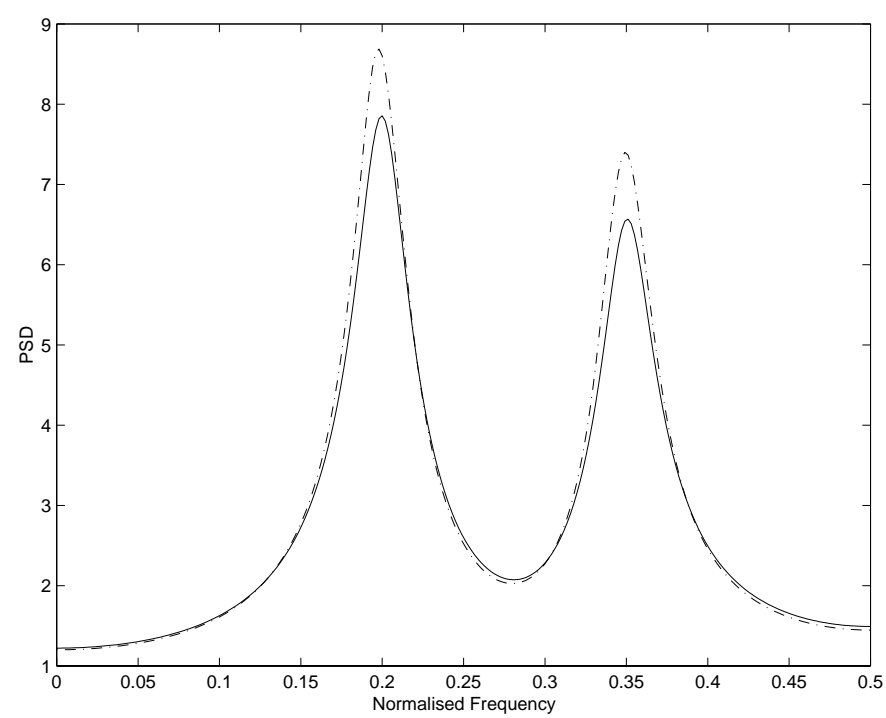

Fig. 3. Continuous line: Theoretical PSD of the simulated signal. Dot-dashed line: Average of the estimated PSD of 110 simulations

of the second step of the proposed algorithm for the estimation of the covariance matrix $Q$, and the third one estimates the performance of the whole algorithm in recovering the parameters.

- The first simulation aims to analyse the effect of modes correlation on the PSD when $L=2$. The poles of the AR models have both modulus 0.9 and angle $\pm 2 \pi 0.25$, $\pm 2 \pi 0.35$. The power of each excitation equals $Q_{11}=$ $Q_{22}=2$ and the three cases: $Q_{12}=0, r_{12}=-3 / 4$, $r_{12}=3 / 4$ have been studied. The three PSD calculated using (15) are given in Fig. 1. The effect of the cross-correlation is clearly visible on the plot but it must be emphasized that this effect disappears when the two modes move away from each other. As previously noticed, the existence of a correlation will affect the measured value of the rotational splitting. 
This result confirms the insight given by the parametric approach for the cross-correlation estimation.

- The purpose of the next simulation is to study the convergence of $\hat{Q}^{[k]}$ and the dependence on the initial values. The poles of the AR models have modulus 0.9 and angle $\pm 2 \pi \cdot 0.15, \pm 2 \pi .0 .35$ and are assumed to be known. $N=200$ samples of the process $y_{n}$ have been generated with $Q_{11}=Q_{22}=2$ and $Q_{12}=1$. Figure 2 represents 10 iterations of (15) when the initial condition equals $\hat{Q}^{[0]}=\alpha I, \alpha=0.5,1,1.5,2,2.5$ and 3. The smoothing has been performed with $R=0.01$. This simulation confirms the correct convergence of the algorithm.

The Cramér Rao bounds (Stoica \& Moses 1997), i.e. the asymptotic variance of the estimated value of $Q$, have been computed for this simulation, see Appendix A. They equal 0.0968 for $Q_{11}, 0.1185$ for $Q_{12}$ and 0.0968 for $Q_{22}$.

- The last simulation of this section evaluates the overall performance of the algorithm. The poles of the model are $0.9 \exp ( \pm j 2 \pi 0.2), 0.87 \exp ( \pm j 2 \pi 0.35)$. The excitation covariance is $Q_{11}=2, Q_{22}=1, Q_{12}=0, R=0.1$ and $N=500.110$ independent realisations of the signal (3) have been generated using these parameters. For each realisation, the poles have been estimated using an autocorrelation matrix of order $M=20$ and the result has been used for the estimation of the excitation covariance. The EM algorithm has been initialised with $\hat{Q}^{[0]}=1.5 I$ and stopped after 100 iterations. Note that for the GOLF signal (next paragraph), the algorithm is performed with 1000 iterations.

The mean and standard deviation of $\hat{Q}$ and $\hat{r}_{12}$ estimated from the 110 computed simulations are:

$\operatorname{mean}[\hat{Q}]=\left(\begin{array}{ll}1.922 & 0.010 \\ 0.010 & 0.977\end{array}\right)$

$\operatorname{std}[\hat{Q}]=\left(\begin{array}{cc}0.228 & 0.272 \\ 0.272 & 0.162\end{array}\right)$,

mean $\left[\hat{r}_{12}\right]=-0.006, \operatorname{std}\left[\hat{r}_{12}\right]=0.198$.

It can be seen that the algorithm does not introduce any bias in the estimation of the covariance matrix $Q$. Even if these estimates do not asymptotically achieve the Cramér-Rao lower bound due to the fact that the dynamical parameters are not estimated by the maximum likelihood method, these results confirm the good performance of the proposed method for the estimation of correlations. Moreover, this performance could be increased by a larger number of iterations. Figure 3 shows that the averaged PSD of the simulations is close to the original one. It has been checked also that the estimates of $Q$ do not depend substantially on the estimates of $F$.
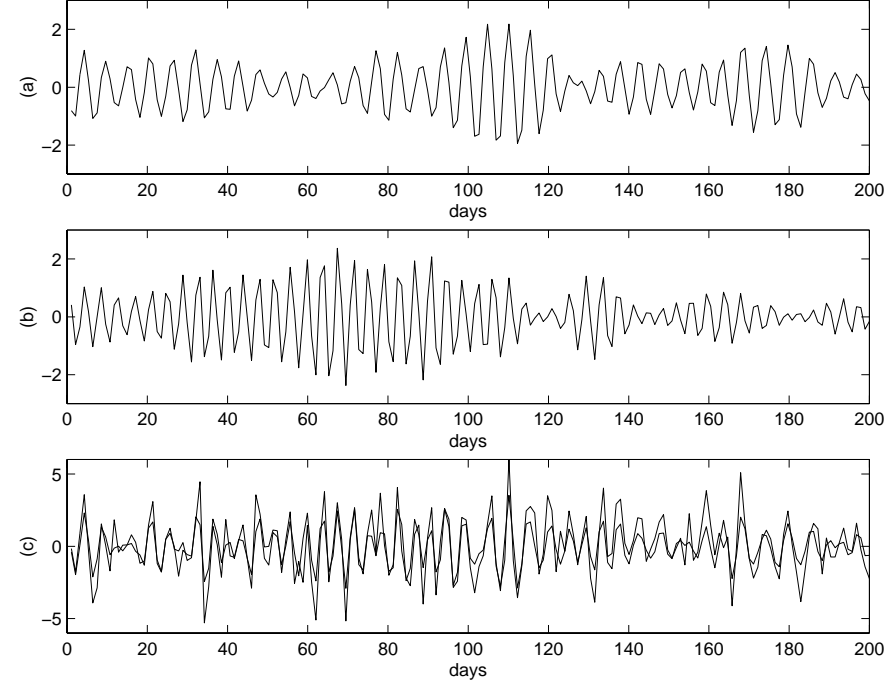

Fig. 4. Band [1746, 1752] $\mu \mathrm{Hz}$. a) and b) reconstructed signal of the first and second mode. c) GOLF signal and smoothed signal

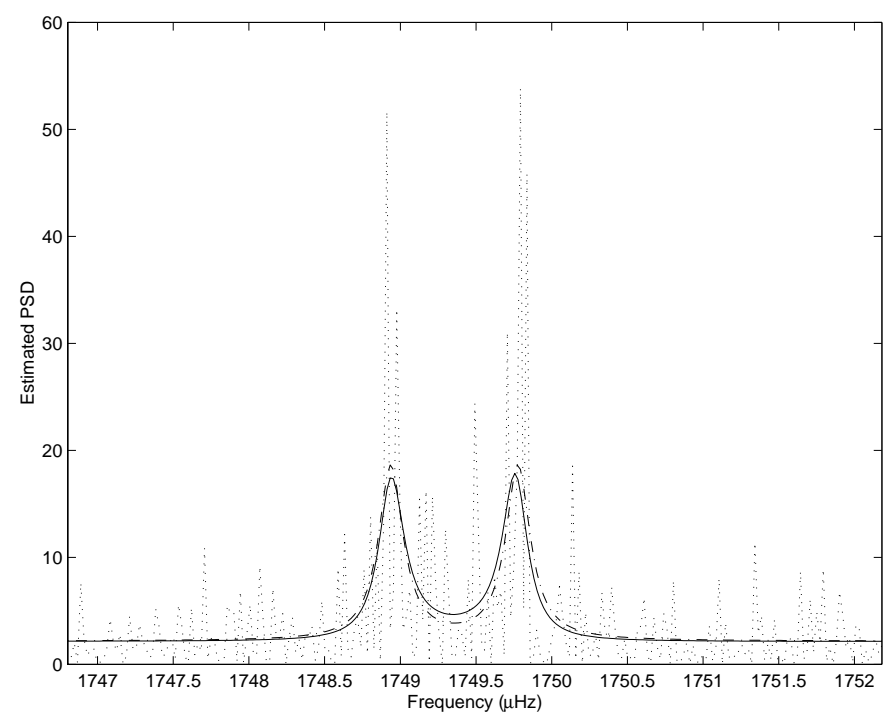

Fig. 5. Estimated PSD. Continuous line (-): proposed method, dashed line $(--)$ : fit of Lorentzian, dotted line $(\cdots)$ periodogram

\subsection{GOLF signal}

The proposed estimation technique has been applied to GOLF data. The data set corresponds to one and half year of continuous observations. The signal corresponding to each analysed frequency band has been demodulated and decimated by direct and inverse Fourier transforms of a 581000 point GOLF signal sampled every 80 seconds.

We analysed two frequency ranges containing $l=1$ modes: the $n=11$ and $n=12$. The first band is [1746, 1752] $\mu \mathrm{Hz}$. The corresponding decimated signal contains $N=503$ points. The poles estimated using an autocorrelation matrix of order $M=10$ are $0.941 \exp ( \pm j 2 \pi 0.198)$ and $0.942 \exp ( \pm j 2 \pi 0.275)$. The estimated value of $R$ is 


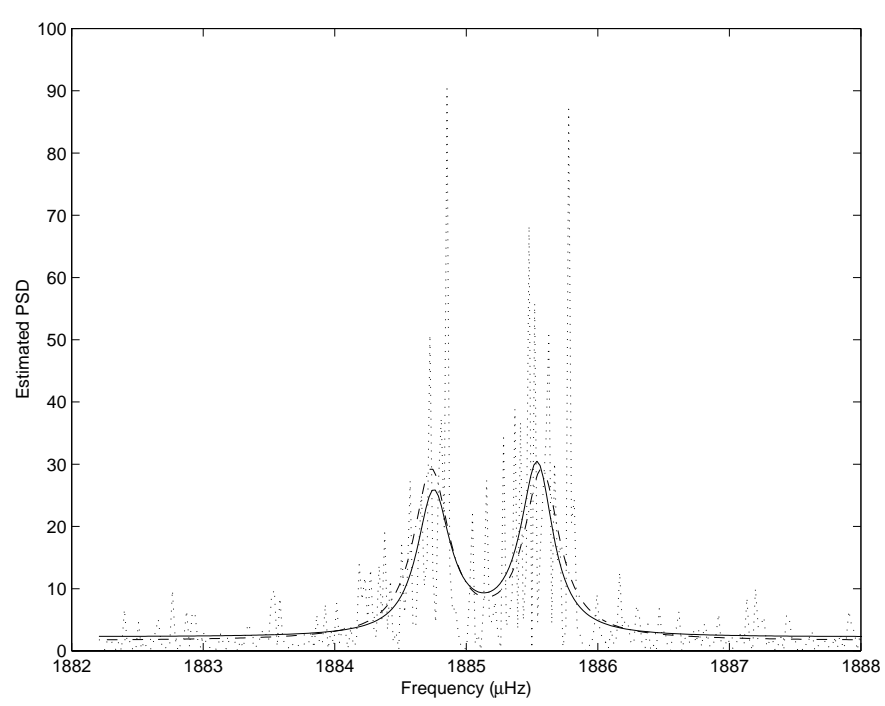

Fig. 6. Estimated PSD. Continuous line (-): proposed method, dashed line $(--)$ : fit of Lorentzian, dotted line $(\cdots)$ periodogram

2.1. The iterations of (15) have been initialised with $\hat{Q}^{[0]}=0.5 I$.

The estimated value of $Q$ after 1000 iterations $\left(\left\|\hat{Q}^{[1000]}-\hat{Q}^{[999]}\right\|_{2} \approx 10^{-6}\right)$ is:

$\hat{Q}=\left(\begin{array}{rr}0.180 & -0.100 \\ -0.100 & 0.193\end{array}\right)$

and $\hat{r}_{12}=-0.533$. Figures $4 \mathrm{a}, 4 \mathrm{~b}$ represent the second and fourth components of the smoothed stated vector at the last iteration, i.e. the estimated signal corresponding to each mode. Figure 4c, represents the corresponding GOLF signal its estimated smoothed version.

The second band is $[1882,1888] \mu \mathrm{Hz}$. The corresponding decimated signal contains $N=559$ points. The poles estimated using an autocorrelation matrix of order $M=$ 10 are $0.922 \exp ( \pm j 2 \pi 0.212)$ and $0.925 \exp ( \pm j 2 \pi 0.278)$. The estimated value of $R$ is 2 . The iterations of (15) have been initialised with $\hat{Q}^{[0]}=0.6 I$. The estimated value of $Q$ after 1000 iterations:

$\hat{Q}=\left(\begin{array}{rr}0.490 & -0.108 \\ -0.108 & 0.570\end{array}\right)$

and $\hat{r}_{12}=-0.204$.

Finally we analysed the triplet $l=2$ in the band [1942, 1950] $\mu \mathrm{Hz}$, with $N=745$. The poles are estimated using an autocorrelation matrix of order $M=20$. In order to take into account the a priori information on the width of the modes, the moduli of the estimated poles have been replaced by the mean of the three estimated moduli, that is all the three modes are forced to have approximately the same width. The corresponding values are: $0.924 \exp ( \pm j 2 \pi 0.188), 0.925 \exp ( \pm j 2 \pi 0.228)$ and $0.929 \exp ( \pm j 2 \pi 0.295)$. The estimated value of $R$ is 2.4 .

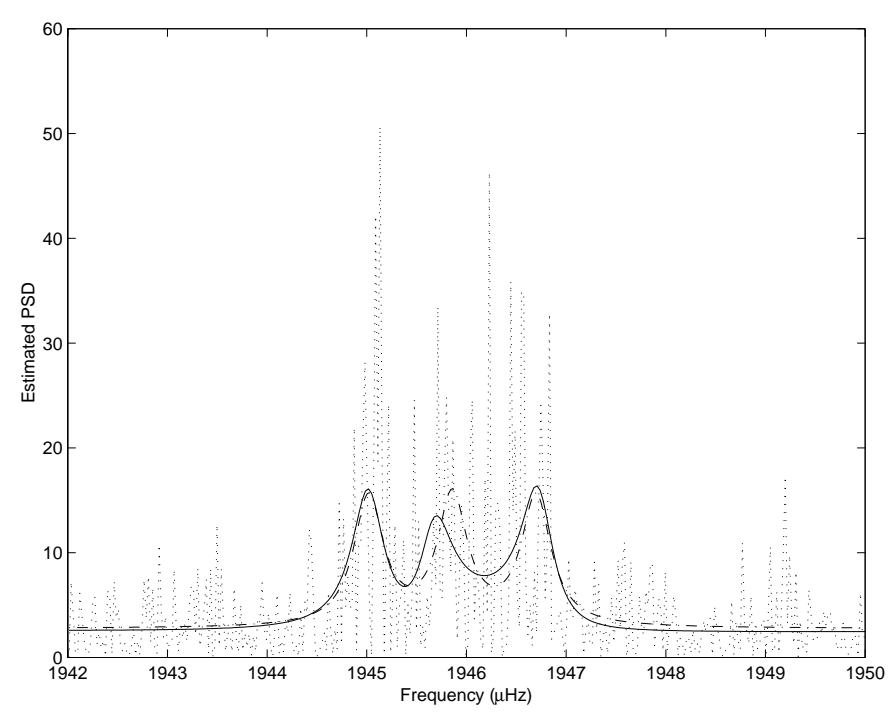

Fig. 7. Estimated PSD. Continuous line (-): proposed method, dashed line $(--)$ : fit of Lorentzian, dotted line $(\cdots)$ periodogram

The iterations of (15) have been initialised with $\hat{Q}^{[0]}=$ $0.3 I$. The estimated value of $Q$ after 1000 iterations is:

$$
\begin{aligned}
& \hat{Q}=\left(\begin{array}{rrr}
0.225 & 0.095 & -0.135 \\
0.095 & 0.169 & -0.156 \\
-0.135 & -0.156 & 0.265
\end{array}\right) \\
& \text { and } \hat{r}_{12}=0.489, \hat{r}_{13}=-0.554 \hat{r}_{23}=-0.737 .
\end{aligned}
$$

In Figs. 5, 6, 7 the estimated PSD are compared to the PSD assuming uncorrelation of the modes and fitting Lorentzian to the periodogram by maximum likelihood criterion, (Fierry-Fraillon et al. 1998).

\section{Conclusion}

The method that we developed has been validated both on simulated and real data. The difficulty of optimisation has been overcome, and we are able to measure the correlation coefficient and the modes parameters. An estimation of the uncertainties of such measurements can be obtained by the Cramér-Rao bound. Although very preliminary, the first results on the few analysed modes show a significant anti-correlation in the excitation of modes with same geometry, but opposite phase. If confirmed, this could have consequences on our view of the excitation mechanism. For instance, it would definitively rule out the possibility of self-excitation of these modes. We have also demonstrated that a correlation on the excitation of split modes affects the shape of the power spectrum and modifies value of the rotational splitting. Such an effect should be measured in order to recover the true rotation profile. More generally, parametric representation is perfectly adapted for the analysis of the helioseismic data and could be used not only for correlation estimation, but also for other purposes like measurement of asymmetries or frequency variation. 
Reliable results have been obtained on a pair of modes, allowing the estimation of excitation correlation between $l=1, m= \pm 1$, or between two $l=0$ modes. For more complicated patterns, we will probably need to constrain the dynamical parameters estimation (e.g. impose the same width and the same separation in triplets $l=2$, as is generally done in Lorentz fitting procedure). Our evaluation of the uncertainties on the measured correlation has also to be improved, in particular to take into account the uncertainties on the dynamical parameters of the modes.

Up to now, the method is only valid for continuous data, and therefore can be applied to GOLF data. This work is still to be done, when the difficulty in the treatment of triplets or quadruplets will be solved. In the future, we would like to improve our procedure in order to be able to analyse non-continuous data such as IRIS or BiSON. The difficulty lies in the filtering of the data, necessary to isolate the considered modes. Some solutions to this problem are presently envisioned.

\section{Appendix A: Computation of $R_{y, M}(\theta, Q)$}

The autocovariance $y_{n}, r_{y}(m)$, equals the sum of the crosscovariances of the modes: $r_{y}(m)=\sum_{i j=1}^{L} E\left[x_{n}^{i} x_{n+m}^{j}\right]+$ $\sigma^{2} \delta(m)=\sum_{i j=1}^{L} r_{i j}(m)+\sigma^{2} \delta(m)$. If $i=j, r_{i j}(m)$ is the autocovariance of an autoregressive process and can be computed inverting the Yule Walker equation (Stoica \& Moses 1997):

$$
\begin{aligned}
& \left(\begin{array}{ccc}
1 & a_{1}^{i} & a_{2}^{i} \\
a_{1}^{i} & 1+a_{2}^{i} & 0 \\
a_{2}^{i} & a_{1}^{i} & 1
\end{array}\right)\left(\begin{array}{l}
r_{i i}(0) \\
r_{i i}(1) \\
r_{i i}(2)
\end{array}\right)=Q_{i i}\left(\begin{array}{l}
1 \\
0 \\
0
\end{array}\right), \\
& \forall q \geq 3, \quad r_{i i}(q)=-a_{1}^{i} r_{i i}(q-1)-a_{2}^{i} r_{i i}(q-2) .
\end{aligned}
$$

If $i \neq j$, multiplying Eq. (2) for $k=i$ by $x^{j}(n)$ and $x^{j}(n+1)$ and multiplying Eq. (2) for $k=j$ by $x^{i}(n-q)$, $q \geq 2$, and taking the expectation, gives:

$$
\begin{aligned}
& \left(\begin{array}{cccc}
1 & a_{1}^{i} & a_{2}^{i} & 0 \\
0 & 1 & a_{1}^{i} & a_{2}^{i} \\
a_{2}^{j} & a_{1}^{j} & 1 & 0 \\
0 & a_{2}^{j} & a_{1}^{j} & 1
\end{array}\right)\left(\begin{array}{c}
r_{i j}(0) \\
r_{i j}(1) \\
r_{i j}(2) \\
r_{i j}(3)
\end{array}\right)=Q_{i j}\left(\begin{array}{c}
1 \\
-a_{1}^{j} \\
0 \\
0
\end{array}\right), \\
& \forall q \geq 4, \quad r_{i j}(q)=-a_{1}^{j} r_{i j}(q-1)-a_{2}^{j} r_{i j}(q-2) .
\end{aligned}
$$

These equations show that $Q_{i j}$ can be factored out in the cross-covariance matrix between $x^{i}(n)$ and $x^{j}(n)$ and then $R_{y, M}(\theta, Q)$ is a linear combination of the $Q_{i j}$.

This result allows a simple computation of the CramérRao bound for the matrix $Q$ using the simplified expression of the Fisher information matrix for a Gaussian distribution (Stoica \& Moses 1997).

\section{Appendix B: Fixed interval smoother equations}

The smoothed states for the model (13) are given by the following two recursions:
Kalman filter (forward recursion)

$$
\begin{aligned}
& K_{k}=P_{k / k-1} H^{t}\left(H P_{k / k-1} H^{t}+\sigma^{2}\right) \\
& \hat{\boldsymbol{X}}_{k / k}=\hat{\boldsymbol{X}}_{k / k-1}+K_{k}\left(y_{k}-H \hat{\boldsymbol{X}}_{k / k-1}\right) \\
& \hat{\boldsymbol{X}}_{k+1 / k}=F \hat{\boldsymbol{X}}_{k / k} \\
& P_{k / k}=\left(I-K_{k} H\right) P_{k / k-1} \\
& P_{k, k-1 / k}=\left(I-K_{k} H\right) F P_{k-1 / k-1} \\
& P_{k+1 / k}=F P_{k / k} F^{t}+G Q G^{t} .
\end{aligned}
$$

The recursion is initialised with: $\hat{\boldsymbol{X}}_{0 /-1}=0$ and $P_{0 /-1}=$ $P_{0}$ where the covariance of the state $P_{0}$ verifies $P_{0}=$ $F P_{0} F^{t}+G Q G^{t}$.

Fixed interval smoother (backward recursion)

$$
\begin{aligned}
& \Gamma_{k}=P_{k / k} F^{t} P_{k+1 / k}^{-1} \\
& \hat{\boldsymbol{X}}_{k / N}=\hat{\boldsymbol{X}}_{k / k}+\Gamma_{k}\left(\hat{\boldsymbol{X}}_{k+1 / N}-\hat{\boldsymbol{X}}_{k+1 / k}\right) \\
& P_{k / N}=P_{k / k}+\Gamma_{k}\left(P_{k+1 / N}-P_{k+1 / k}\right) \Gamma_{k}^{t} \\
& P_{k, k-1 / N}=P_{k, k-1 / k}+\left(P_{k / N} P_{k / k}^{-1}-I\right) P_{k, k-1 / k} .
\end{aligned}
$$

The recursion is initialised with: $\hat{\boldsymbol{X}}_{N / N}$ and $P_{N / N}$.

Acknowledgements. The authors are especially gratefull to John Leibacher for reading the manuscript and to Bernard Gelly for providing the Lorentzian fits. We wish to thank the GOLF team for providing us the data samples we used to test our method.

\section{References}

Anderson B.D., Moore J., 1979, Optimal Filtering. Prentice-Hall

Balmforth N., 1992, MNRAS 255, 639

Baudin F., Gabriel A., Gibert D., Pallé P., Règulo C., 1996, A\&A 311, 1024

Brillinger D., 1981, Time Series: Data Analysis and Theory. McGraw-Hill

Chaplin W., Elsworth Y., Howe R., et al., 1997, MNRAS 287,51

Dempster A., Laird N., Rubin D., 1977, J. Royal Statist. Soc. Ser. B 39, 1

Deng L., Shen X., 1997, EURASIP Sig. Proc. 57, 65

Fierry-Fraillon D., Gelly B., Schmider F.-X., et al., 1998, A\&A 333, 362

Foglizzo T., Garcìa R., Boumier P., et al., 1998, A\&A 330, 341

Gabriel A., Grec G., Charra J., et al., 1995, Solar Phys. 162,61

Gabriel M., 1995, A\&A 299, 245

Gavryusev V., Gavryuseva E., 1997, Solar Phys. 172, 27

Kumar P., 1997, in Provost J. and Schmider F.-X. (eds.), Proc. Symp. IAU 181: Sounding Solar and Stellar Interiors. Kluwer Academic Publishers, p. 287 
Lazrek M., Baudin F., Bertello L., et al., 1997, Solar Phys. Roxburgh I., Vorontsov S., 1995, MNRAS 272, 850 175,227

Moon T., 1996, IEEE Signal Processing Magazine, pp. $47-60$

Sage A., Melsa J., 1971, System Identification, Vol. 80. Academic Press

Peter Goldreich N.M., Kumar P., 1994, A\&A 424466

Stoica P., Moses R., 1997, Introduction to Spectral

Priestley M.B., 1994, Spectral Analysis and Time Series. Toutain T., Appourchaux T., 1994, A\&A 289, 649 Academic Press, 8th edition 